\title{
DISEÑO DE UN SISTEMA PARA LA EVALUACIÓN DE LA EFECTIVIDAD DEL LANZAMIENTO EN BOCCIA
}

\author{
Julie Joan Acevedo Ramírez ${ }^{1}$, Luz Amelia Hoyos Cuartas ${ }^{2}$, Rosa Elena Mendivelso Leal ${ }^{3}$ \\ ${ }^{1}$ Especialista en Discapacidad, Educación Física, Recreación y Deporte: Adaptados. Docente Investigadora, \\ Línea actividad física y discapacidad. Julie.acevedo@cenda.edu.co \\ 2 Doctora en Ciencias de la Actividad Física y el Deporte. Coordinadora Especialista en Discapacidad, \\ Educación Física, Recreación y Deporte: Adaptados. luzahoyos@yahoo.com \\ ${ }^{3}$ Especialista en Comunicación Aumentativa y Alternativa. Docente Investigadora, Línea actividad física y \\ discapacidad, Licenciatura en Educación Física, Recreación y Deporte. lic.rosamendivelso@gmail.com \\ 1,2,3 Corporación Universitaria CENDA. Bogotá-Colombia
}

\section{RESUMEN}

Objetivo: diseñar un sistema integrado que permita evaluar de forma objetiva la efectividad de los fundamentos técnicos del lanzamiento en Boccia, con el propósito de transformar la práctica y el entrenamiento de este deporte. Metodología: Esta investigación corresponde a un diseño tecnológico basado en el desarrollo de un test y un software, con pruebas de validación y pilotaje en un grupo experimental resultados, se desarrolló un sistema integrado que permiten determinar el nivel de efectividad de cada deportista y ubicarlo en una categoría: baja, regular, buena o excelente, para cada una de las variables, de acuerdo a su desempeño. Conclusión: El sistema permite identificar de forma objetiva la efectividad del lanzamiento discriminando el desempeño de cada uno de sus fundamentos técnicos, igualmente permite crear un esquema de juego para competencia, ubicando a los deportistas en el box más estratégico y brinda una herramienta de control para los entrenadores.

Palabras clave: Boccia, efectividad de los fundamentos técnicos, test, diseño de software.

Recibido: 18 de mayo de 2018. Aceptado: 15 de noviembre de 2018

Received: May 18th, 2018. Accepted: November 15th, 2018

\section{DESIGN OF A SYSTEM FOR THE EVALUATION OF THE EFFECTIVENESS OF THE THROW IN BOCCIA.}

\begin{abstract}
Objective: design an integrated system that allows an objective evaluation of the effectiveness of the technical foundations of the pitching in Boccia, with the purpose of transforming the practice and training of this sport. Methodology: This research corresponds to a technological design based on the development of a test and a software, with validation and piloting tests performed on an experimental group. Results, an integrated system was developed to determine the level of effectiveness of each athlete and place it in a category: low, regular, good or excellent, for each of the variables, according to their performance. Conclusion: The system allows to objectively identify the effectiveness of the pitch discriminating the performance of each one of its technical fundamentals, it also allows to create a game scheme for competition, placing athletes in the most strategic box and provides a control tool for coaches.
\end{abstract}

Keywords: Boccia, effectiveness of technical fundamentals, test, software design

Cómo citar este artículo: J. J. Acevedo Ramírez, L. A. Hoyos Cuartas, R. H. Mendivelso Leal, "Diseño de un sistema para la evaluación de la efectividad del lanzamiento en Boccia”, Revista Politécnica, vol. 14, no. 27 pp.57-67, 2018. https://doi.org/10.33571/rpolitec.v14n27a6 


\section{INTRODUCCIÓN}

El deporte paralímpico surge como práctica deportiva adaptada con fines terapéuticos para personas con discapacidad, su formalización se le atribuye al doctor Guttman, quien implemento un proceso de rehabilitación para los veteranos de guerra, en la cuidad de Mandeville (Inglaterra), en 1950. Actualmente es considerado como deporte de alto rendimiento y se rige bajo los mismos parámetros del deporte olímpico, Ruiz, [1]. El Boccia es un deporte para personas con discapacidad física, y se caracteriza por el alto nivel de precisión que se requiere para los lanzamientos, ingresa como deporte oficial en los Juegos Paralímpicos de Seúl 1988, sin embargo, sus inicios se remontan al siglo XVI en Grecia y fue retomado en los años 70 por los países nórdicos con el fin de adaptarlo a las personas con discapacidad. (Boccia International Sport Federation, [2]

En sus inicios fue practicado por atletas con parálisis cerebral, condición que es definida por Rosell, Camats, y Basil, [3] como un trastorno persistente del movimiento y de la postura, causado por una lesión no progresiva del sistema nervioso central. En la actualidad incluye también, a deportistas que tienen otras discapacidades, en las que se afectan las habilidades motoras; personas con lesión medular, múltiples discapacidades y distrofia muscular degenerativa; esta distrofia es entendida como una miopatía de carácter genético causado por la ausencia de proteína distrofina y su característica más relevante es la pérdida progresiva de fuerza y de masa musculatura, Silva, Fonseca, Mateus, Contreras, y Restrepo, [4]. Este deporte implica en el jugador un dominio importante de los fundamentos técnicos, que inciden en el desempeño deportivo, para Martin, Carl, y Lehnertz, [5] "ha de contemplarse el aspecto de su efecto integrador, pues en la medida que las capacidades coordinativas y condicionales se puedan trasformar en rendimientos de competición depende en gran medida del nivel de destreza técnica en el deporte" (p.49). Collazo [6] afirma que la técnica, es considerada como un patrón motor, que va siendo modificado de manera progresiva, mientras que Barrios y Ranzola [7], afirman que la técnica es una cadena de movimientos organizados que permite el cumplimento de una tarea motora acorde a lo exigido. Barrios y Ranzola, [7] consideran que en un deporte de arte y precisión como el Boccia es de vital importancia evaluar y controlar de manera rigurosa tanto la biomecánica del gesto deportivo como la precisión al momento de poner en práctica los fundamentos técnicos y más aún cuando es practicado bajo el concepto de deporte de altos logros; Harre [8] afirma que "el control del rendimiento, es el registro del rendimiento de cada uno de los deportistas mediante la medición, el conteo, la observación y evaluación en un deporte o disciplina, con el objetivo de constatar el efecto entrenador de cada una de las cargas o el estado de entrenamiento de cada deportista" (p. 60) . En el deporte del Boccia es prioritario diseñar un sistema de evaluación de los fundamentos técnicos, con el fin de realizar un control del entrenamiento y así encaminar los procesos deportivos de cada atleta. Para Green [9], las pruebas son fundamentales en la medición de los factores del entrenamiento. "Estos factores una vez medidos pueden ser evaluados para desarrollar estrategias de entrenamiento apropiadas que ayuden a superar cualquier tipo de desventaja" ( $p$. 21).

Finalmente dentro de los antecedentes de estudios, se pueden referir el trabajo de Avila, y Moreno [10] que mide la percepción y la actividad neuromuscular en deportistas de Boccia con parálisis cerebral, y la investigación de Ramón y Ortega [11] que analizó los efectos del entrenamiento deportivo en relación con la función motora gruesa y la independencia funcional en niños y jóvenes con parálisis cerebral. En la misma perspectiva, y en relación con el desarrollo de software especializados para los deportes, Bustamante y Burrillo [12], afirman que: "en el ámbito del rendimiento deportivo, también existe una creciente necesidad de colección de datos que aporten evidencia empírica sobre la compleja realidad a la que se refieren" (p.72). A partir de estos antecedentes, se identifica que es urgente diseñar y aplicar un sistema de evaluación de la efectividad de los fundamentos técnicos del lanzamiento de Boccia, en espacios específicos de la zona de juego, dividiéndolo en espacios más reducidos para una evaluación más rigurosa. Este sistema de evaluación requiere ser complementado con un software que sistematice y analice la información de cada jugador.

Los fundamentos técnicos entendidos como el conjunto de procesos que se asimilan con el ejercicio, y permiten realizar una tarea de forma racional, económica y con la máxima eficacia Manno [13]. En el test diseñado para este estudio, 
se consideran los fundamentos técnicos del Boccia: salida, arrimar, apoyar, romper montar, realizando este análisis desde el box 1 al box 6 según la división de juego de cada uno de los deportistas (individual, parejas o equipos), y ajustando cada lanzamiento a las exigencias del tiempo reglamentado según la clasificación funcional de cada deportista. Este test evalúa la efectividad de cada deportista, entendiendo la efectividad como la relación que existe entre los resultado alcanzado bajo condiciones reales y resultados esperados en unas condiciones óptimas de juego, Lozada, Casal y Ardá, [14]. Por su parte Acero [15], la considera como la comparación entre el número total de intentos de una técnica deportiva y el número de técnicas realizadas de forma correcta, que se asemejan al modelo ideal. Dicha efectividad es la capacidad de repetir acertadamente una técnica o de mantener una estabilidad de ejecución. De esta manera, el test permite medir el nivel de efectividad y evidenciar las fortalezas y debilidades de los deportistas y la ubicación estratégica para competencia realizando una evaluación de los fundamentos técnicos y proponiendo esquemas de competición que tienen en cuenta como lo plantea Acero [16] la variabilidad para ser modificados o ajustados cada vez y así poder conseguir el objetivo final.

El objetivo del presente estudio fue diseñar un sistema integrado que permita evaluar de forma objetiva la efectividad de los fundamentos técnicos del lanzamiento en Boccia, con el propósito de transformar la práctica y el entrenamiento de este deporte.

\section{MATERIALES Y METODO}

En primera instancia se diseñó un test que a partir de la observación de 5 variables técnicas fundamentales: salida, arrimar, apoyar, romper montar, las cuales permiten determinar la efectividad del lanzamiento del deportista. A su vez el terreno de juego se dividió en 12 zonas o cuadrantes hacia donde se debían orientar los lanzamientos. Para la calificación del desempeño del deportista en cada una de las variables se utilizó una escala tipo Likert de 5 niveles con la siguiente ponderación:

0 puntos: no cumplió el objetivo

1 punto: Esta en el rango pero no en la trayectoria.

2 puntos: está en la trayectoria a más de $4 \mathrm{~cm}$.
3 puntos: está en la trayectoria pero con distancia de menos de $4 \mathrm{~cm}$.

4 puntos: cumple el objetivo.

Posteriormente se creó un software para analizar puntuar y almacenar la información obtenida.

Para la implementación, ajuste y validación del sistema de evaluación de la efectividad en el lanzamiento, se realizó un trabajo experimental con 13 deportistas, (4 mujeres y 9 hombres) entre los 10 y 33 años de edad, con parálisis cerebral perteneciente a la selección de Boccia del departamento de Cundinamarca, con quienes se efectuaron las pruebas y validaciones en los diferentes momentos de la concepción y desarrollo del sistema.

\section{RESULTADOS}

Se logró consolidar un sistema que permite determinar la efectividad del lanzamiento en el deporte de Boccia. Se diseñó un test que permite evaluar de forma objetiva 5 aspectos fundamentales de la técnica del lanzamiento en el deporte de Boccia y un software que almacena la información y genera los reportes de puntuación de cada deportista.

\section{Descripción técnica del test}

Cada jugador se ubica en un box según su clasificación funcional y la división de juego que se quiere evaluar. Cuando la división del juego es individual, los deportistas se sitúan en el box 3 y 4 , cuando es por parejas, en los box 2, 3, 4, 5 y cuando es por equipos del box 1 al 6 . Se le da al deportista la diana (Boccia blanca) y la Boccia respectiva al color que corresponda según el box de ubicación, (rojo si está en el box 3 o azul si está en el box 4) cuando el evaluador muestre la paleta al jugador, se pone en marcha el cronometro y se detiene cuando la Boccia esté totalmente quieta.

El deportista tendrá un tiempo límite para ejecutar los lanzamientos que se deben realizar según la ubicación del deportista según su clasificación funcional establecida en el reglamento. Si se encuentra entre el box 1 al 3 se inicia lanzando hacia las zonas I1 hasta el I4, luego a las zonas C1 hasta C4 y finalmente a las zonas D1 a D4. Si el deportista inicia entre el box 4 al 6 el orden de lanzamiento se realiza en el sentido contrario, es decir se parte de la zonas $D$ para finalizar en las 
zonas I. En la figuras 2 y 3 se realiza una representación gráfica del orden de las zonas según el box donde se inicie.

Para la calificación de cada uno de los lanzamiento se diseñaron dos aros (en material plástico de $1 \mathrm{~cm}$ de ancho y $0.1 \mathrm{ml}$ de alto) uno de $50 \mathrm{~cm}$ (rojo) y otro de $16 \mathrm{~cm}$, de diámetro (Azul), como se muestra en la figura 1.

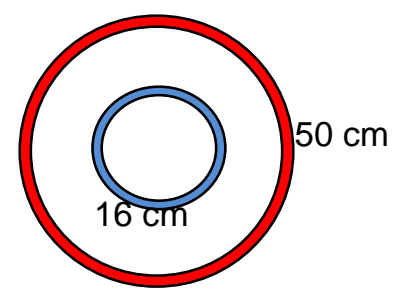

Figura 1. Aro de evaluación

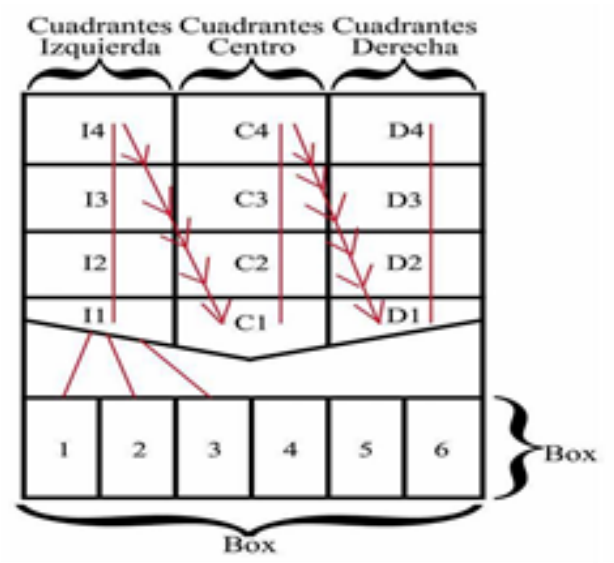

Figura 2: Dirección de los lanzamientos deportistas está en el box 4-6

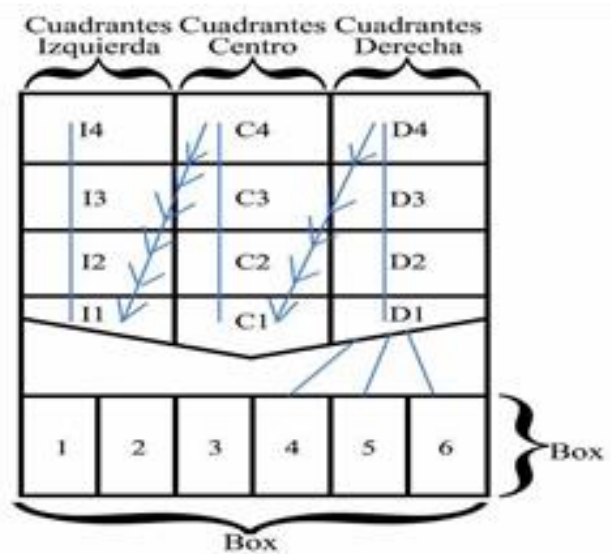

Figura 3: Dirección de los lanzamientos cuando los cuando los deportistas están en el box 1-3
Fundamento técnico salida:

Descripción: Se entiende por fundamento técnico salida cuando el jugador lanza la Boccia diana (color blanco) y seguidamente lanza la primera Boccia de color (rojol azul) cuyo objetivo principal es hacer que las dos Boccia lanzadas queden lo más cerca posible.

Cuando la Boccia diana se detiene, se ubican los dos aros que tendrán como centro la Boccia diana. Posteriormente se lanzan cuatro Boccias de color y de acuerdo a su ubicación dentro del perímetro del círculo donde finalice, se califica de la siguiente manera:

Cero puntos, cuando la Boccia de color queda por fuera del perímetro del círculo de $50 \mathrm{~cm}$.

Un punto, cuando la Boccia de color, queda dentro del perímetro de $50 \mathrm{~cm}$, pero no en la dirección que bloque al oponente.

Dos puntos, cuando la Boccia de color, queda dentro del perímetro de $50 \mathrm{~cm}$ y queda bloqueando al oponente.

Tres puntos cuando la Boccia de color, queda dentro del perímetro de $16 \mathrm{~cm}$, pero no en la dirección que bloque al oponente.

Cuatro puntos cuando la Boccia de color, queda dentro del perímetro de $16 \mathrm{~cm}$ y queda bloqueando al oponente.

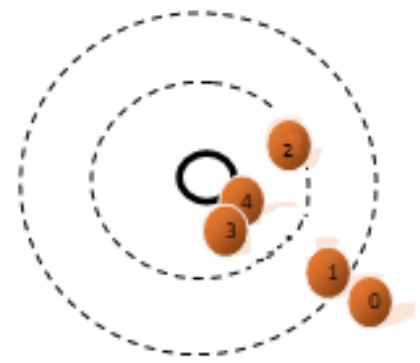

Figura 4: Esquema de evaluación fundamento técnico salida.

Descripción del Fundamento técnico arrimar:

Se entiende por Arrimar cuando en el campo de juego el evaluador ubica la diana en las diferentes zonas en que se divido el terreno. El jugador ejecuta el lanzamiento con la intención de ubicarse lo más cerca posible de la diana. La evaluación se 
realiza de la misma forma como se describió anteriormente para el lanzamiento.

Fundamento técnico apoyar:

El fundamento técnico apoyar, se refiere a la situación de juego cuando la Diana está bloqueada por una Boccia del oponente. El jugador realiza dos lanzamientos y con el primero ubica la Boccia cerca a la diana, de tal forma que sirva de apoyo para la Boccia del segundo lanzamiento. Con el segundo lanzamiento se busca que la Boccia pegue en la Boccia anteriormente lanzada y rebote de tal forma que se impulse para realizar contacto con la diana.

La puntuación se realiza de la siguiente manera:

Cero puntos, cuando fabrica el apoyo con la primera Boccia, pero la segunda Boccia lanzada no hace contacto con la Boccia

Un punto, cuando fabrica el apoyo con la primera Boccia pero la segunda Boccia lanzada rebota, pero se ubica fuera del diámetro de 50 centímetros.

Dos puntos cuando fabrica el apoyo con la primera Boccia y con la segunda Boccia lanzada rebota pero se ubica en el perímetro de $50 \mathrm{~cm}$.

Tres puntos cuando fabrica el apoyo con la primera Boccia y con el segundo lanzamiento la Boccia rebota pero esta se ubica en el diámetro de $16 \mathrm{~cm}$ por detrás de la diana.

Cuatro puntos: cuando fabrica el apoyo con la primera Boccia, y con la segunda Boccia lanzada rebota quedando en total contacto con la Diana.
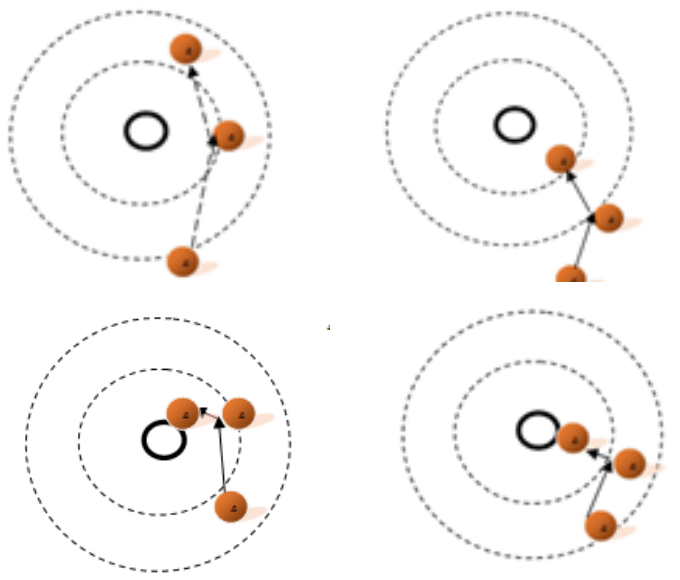

Figura 5: Esquema de evaluación fundamento técnico Apoyar
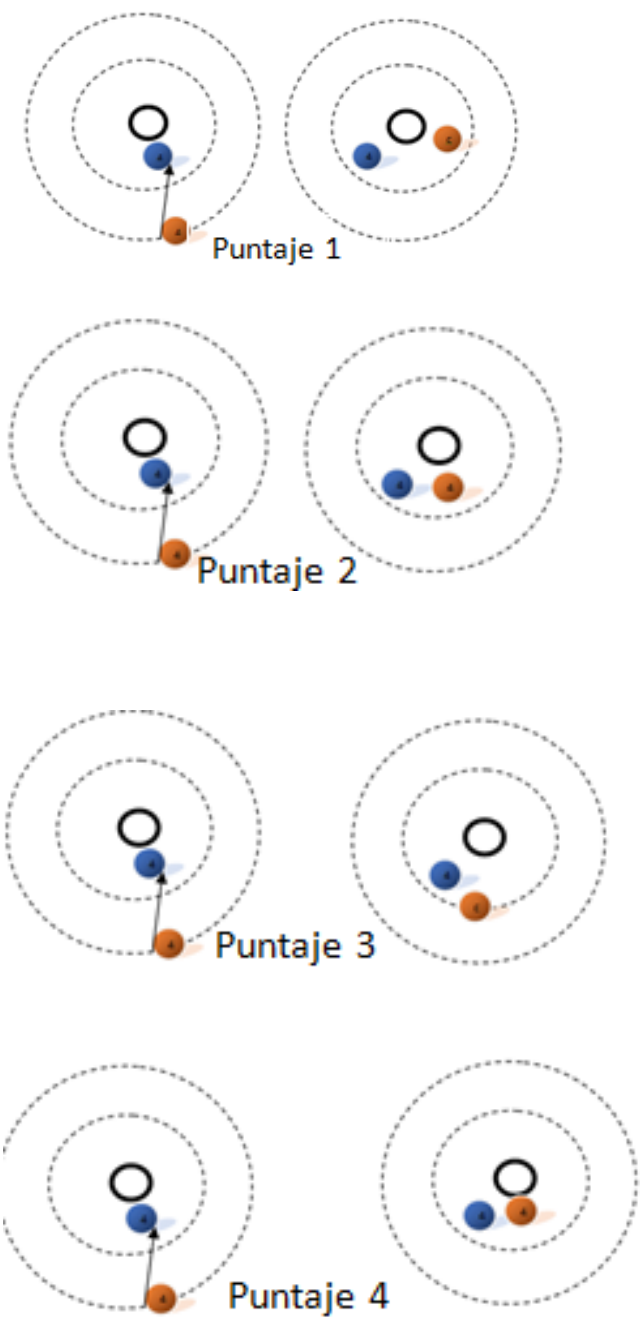

Figura 6. Esquema de evaluación del fundamento técnico romper

Fundamento técnico romper:

Cuando en el campo de juego la diana está en total contacto con una Boccia del oponente el objetivo del lanzamiento es alejar de la diana la Boccia del oponente, aumentando la distancia entre estas dos y logrando ubicar la Boccia lanzada, lo más cerca a la Diana.

La puntuación se asigna de la siguiente manera:

Cero puntos cuando, la Boccia lanzada no queda dentro el perímetro de $50 \mathrm{~cm}$. Ni hace contacto con la Boccia del oponente. 
Uno cuando la Boccia lanzada golpea la del oponente y la aleja de la diana y finaliza en el perímetro de $50 \mathrm{~cm}$, pero no queda bloqueando al oponente

Dos puntos: cuando la Boccia lanzada golpea la del oponente y la aleja de la diana y finaliza en el perímetro de $16 \mathrm{~cm}$ pero no queda bloqueando al oponente.

Tres puntos: cuando la Boccia lanzada golpea la del oponente y la aleja de la diana y finaliza haciendo contacto con la diana, pero no queda bloqueando al oponente.

Cuatro puntos cuando la Boccia lanzada golpea la del oponente y la aleja de la diana y finaliza haciendo contacto con la diana y bloqueando al oponente.

En la figura 6, se muestran dos gráficos en primero mostrará la ubicación inicial y la dirección de la Boccia lanzada y el segundo mostrará la ubicación final de las Boccias.

\section{Fundamento técnico montar}

Se entiende por montar cuando dos o más Boccias están en el campo de juego en contacto con la diana y con el lanzamiento se busca ubicar la Boccia sobre las Boccias que están junto a la diana.

La puntuación se asigna de la siguiente manera:

0 puntos: Cuando la Boccia lanzada se ubica en el perímetro de 50 centímetros sin montarse en las Boccias del oponente.

Un punto Cuando la Boccia lanzada se monta en las Boccias pero cae y se ubica en el perímetro de 50 centímetros.

Dos puntos Cuando la Boccia lanzada se monta en las Boccias pero cae y se ubica en el perímetro de $16 \mathrm{~cm}$.
Tres puntos: cuando la Boccia lanzada se monta encima de las otras Boccias pero no hace contacto con la diana.

Cuatro puntos: cuando la Boccia lanzada se monta encima de las otras Boccias haciendo contacto con la diana.

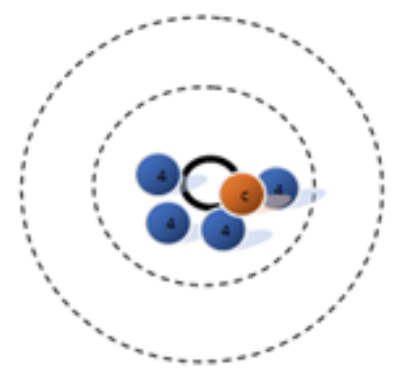

Figura 7. Esquema de evaluación del fundamento técnico Montar

\begin{tabular}{|c|c|c|c|}
\hline Elemento & Test Inicial & Modificación & Test Final \\
\hline 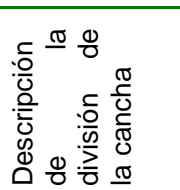 & $\begin{array}{l}\text { La zona de } \\
\text { juego se } \\
\text { dividió en } 50\end{array}$ & $\begin{array}{ll}\text { La zona de } \\
\text { juego } & \text { se } \\
\text { dividió en } 12 \\
\text { cuadrantes }\end{array}$ & $\begin{array}{ll}\text { La zona de } \\
\text { juego } \\
\text { dividió en } 12 \\
\text { cuadrantes }\end{array}$ \\
\hline 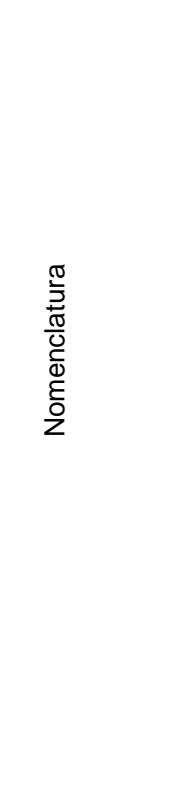 & $\begin{array}{l}\text { Coordenadas } \\
\text { de la A hasta } \\
\text { la I en el } \\
\text { plano vertical } \\
\text { y en el plano } \\
\text { horizontal } \\
\text { desde el } \\
\text { numero } 1 \\
\text { hasta el } 6 .\end{array}$ & $\begin{array}{l}\text { Se le dio } \\
\text { nombres a los } \\
4 \text { cuadrantes } \\
\text { por derecha } \\
\text { llamados (d1, } \\
\text { d2, d3, d4) } \\
\text { cuadrantes } \\
\text { por el centro } \\
\text { (c1, c2, c3, } \\
\text { c4) y } \\
\text { cuadrantes } \\
\text { por izquierda } \\
\text { (i1, i2, i3, i4) } \\
\text { donde i1 está } \\
\text { ubicado al } \\
\text { final de la } \\
\text { zona de juego } \\
\text { y i4 está } \\
\text { ubicada en la } \\
\text { zona más } \\
\text { cerna a los } \\
\text { box. }\end{array}$ & $\begin{array}{l}\text { Se le } \\
\text { designaron } \\
\text { nombres a } \\
\text { los } \\
\text { cuadrantes } \\
\text { teniendo, } 4 \\
\text { por derecha } \\
\text { llamados (d1, } \\
\text { d2, d3, d4) } \\
\text { cuadrantes } \\
\text { por centro } \\
\text { (c1, c2, c3, } \\
\text { c4) y } \\
\text { cuadrantes } \\
\text { por izquierda } \\
\text { (i1, i2, i3 i4) } \\
\text { donde i1 está } \\
\text { ubicado al } \\
\text { inicio de la } \\
\text { zona de } \\
\text { juego y i4 } \\
\text { está ubicado } \\
\text { en la zona } \\
\text { final de } \\
\text { juego. }\end{array}$ \\
\hline 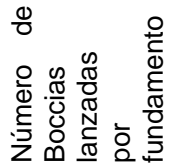 & $\begin{array}{l}6 \text { Boccias } \\
\text { por } \\
\text { fundamento }\end{array}$ & $\begin{array}{l}6 \text { Boccias por } \\
\text { fundamento }\end{array}$ & $\begin{array}{l}4 \text { Boccias por } \\
\text { fundamento }\end{array}$ \\
\hline
\end{tabular}




\begin{tabular}{|c|c|c|c|}
\hline Elemento & $\begin{array}{l}\text { Test } \\
\text { Inicial }\end{array}$ & Modificación & Test Final \\
\hline 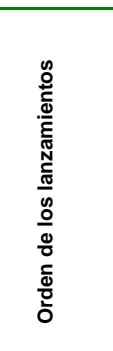 & $\begin{array}{l}\text { Serán } \\
\text { lanzadas } \\
\text { hacia la } \\
\text { primera } \\
\text { coordena } \\
\text { da, que } \\
\text { el } \\
\text { deportista } \\
\text { elige de } \\
\text { manera } \\
\text { aleatoria. }\end{array}$ & $\begin{array}{l}\text { Los lanzamientos se } \\
\text { realizan según la } \\
\text { ubicación del } \\
\text { deportista, si está en el } \\
\text { box } 1 \text { y } 2 \text { inicia con i1 } \\
\text { y finaliza en d4, si el } \\
\text { deportista está en el } \\
\text { box } 3 \text { y } 4 \text { inicia con c1 } \\
\text { y termina con i4 y si el } \\
\text { deportista está en el } \\
\text { box } 5 \text { y } 6 \text { inicia en el } \\
\text { d1 y finaliza en c4. }\end{array}$ & $\begin{array}{l}\text { Los lanzamientos se } \\
\text { realizan según la } \\
\text { ubicación del deportista: } \\
\text { Box } 1,2,3 \text { se inicia } \\
\text { desde el i } 1 \text { hasta el i4 } \\
\text { pasa al c1 hasta el c4 y } \\
\text { sigue en d1 a d4. Si el } \\
\text { deportista inicia en el box } \\
4,5,6 \text { inicia en d1 hasta } \\
\text { d4 pasa a c1 hasta c4 y } \\
\text { sigue de i } 1 \text { a i4. }\end{array}$ \\
\hline 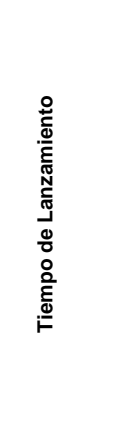 & $\begin{array}{l}\text { No se } \\
\text { definió el } \\
\text { tiempo de } \\
\text { lanzamie } \\
\text { nto }\end{array}$ & $\begin{array}{l}\text { - bc1: tiempo límite de } \\
40 \text { segundos por } \\
\text { Boccia } \\
\text { - bc2: Tiempo límite de } \\
30 \text { segundos por } \\
\text { Boccia } \\
\text { - bc3: Tiempo Límite de } \\
50 \text { segundos por } \\
\text { Boccia. } \\
\text { - Bc4: tiempo límite de } \\
30 \text { segundos por } \\
\text { Boccia. }\end{array}$ & $\begin{array}{l}\text { Se asigna un tiempo } \\
\text { distinto para el } \\
\text { Lanzamiento de acuerdo } \\
\text { a la clasificación } \\
\text { deportiva del jugador. } \\
\text { Bc1: tiempo límite de } 2: 40 \\
\text { para lanzar las } 4 \text { Boccias } \\
\text { Bc2: Tiempo límite de } \\
\text { 2:17 para lanzar las } 4 \\
\text { Boccias } \\
\text { Bc3: Tiempo Límite de } \\
\text { 3:20 para lanzar las } 4 \\
\text { Boccias } \\
\text { Bc4: tiempo límite de 2:17 } \\
\text { para lanzar las } 4 \text { Boccias. }\end{array}$ \\
\hline 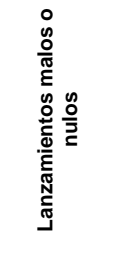 & $\begin{array}{l}\text { No se } \\
\text { contempl } \\
\text { ó registrar } \\
\text { los } \\
\text { Lanzamie } \\
\text { ntos } \\
\text { nulos o } \\
\text { nulos }\end{array}$ & $\begin{array}{l}\text { Las Boccias que no } \\
\text { sean lanzadas o que se } \\
\text { comenta una falta } \\
\text { según reglamento se le } \\
\text { dará puntaje de } 0 .\end{array}$ & $\begin{array}{l}\text { Si el deportista comete } \\
\text { una falta, el lanzamiento } \\
\text { será nulo, (Las faltas } \\
\text { están contempladas en el } \\
\text { reglamento). Las Boccias } \\
\text { que no alcancen a ser } \\
\text { lanzadas se contaran } \\
\text { como lanzamientos malos } \\
\text { con un valor de } 0 \text {. }\end{array}$ \\
\hline 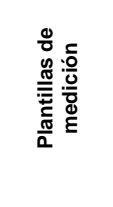 & $\begin{array}{l}\text { No se } \\
\text { contaba } \\
\text { con una } \\
\text { plantilla } \\
\text { de } \\
\text { medición }\end{array}$ & $\begin{array}{l}\text { La plantilla de medición } \\
\text { medía } 40 \text { centímetros } \\
\text { de circunferencia. }\end{array}$ & $\begin{array}{l}\text { La plantilla de medición } \\
\text { se ajustó de } 16 \\
\text { centímetros y otra de } 50 \\
\text { centímetros ya que era } \\
\text { más funcional de acuerdo } \\
\text { a las características del } \\
\text { juego. }\end{array}$ \\
\hline 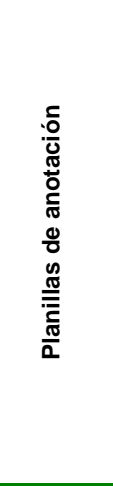 & $\begin{array}{l}\text { Las } \\
\text { planillas } \\
\text { no tenían } \\
\text { grafico de } \\
\text { los } \\
\text { fundament } \\
\text { os técnicos } \\
\text { con } \\
\text { valoración. }\end{array}$ & $\begin{array}{l}\text { Las planillas no tenían } \\
\text { grafico de los } \\
\text { fundamentos técnicos } \\
\text { con valoración. }\end{array}$ & $\begin{array}{l}\text { Se evidencio la } \\
\text { importancia de tener } \\
\text { unificados criterios de } \\
\text { evaluación para la } \\
\text { calificación de cada } \\
\text { fundamento, de tal } \\
\text { manera que las planillas } \\
\text { de registro cuentan con } \\
\text { un dibujo del puntaje que } \\
\text { se le debe dar a cada } \\
\text { fundamento al finalizar el } \\
\text { lanzamiento con una } \\
\text { explicación breve del } \\
\text { gráfico, para que el } \\
\text { evaluador sea más } \\
\text { objetivo al dar la } \\
\text { puntuación. }\end{array}$ \\
\hline
\end{tabular}

El terreno de juego debe cumplir con la especificación en el reglamento estipulado por la: Boccia International Sports Federation (BISFed).

Software
El diseño del software se realiza bajo un ambiente Php y con una base de datos en MysQI, que permite su ejecución en un entorno web lo que indica que se puede utilizar en cualquier parte del mundo y en cualquier dispositivo que tenga conexión a internet.

En la figura 8 se muestra la ventana inicial del programa donde se da clic derecho, en "acceder a la aplicación" como lo indica la flecha negra.

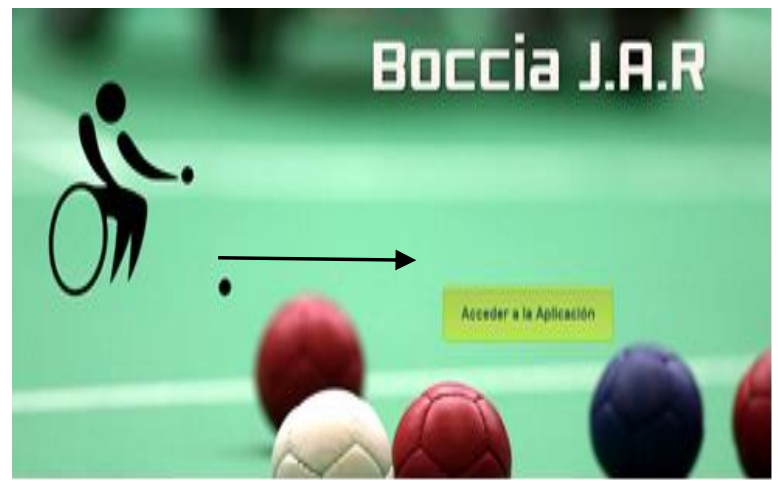

Figura 8. Ventana Inicial de acceso.

Una vez se accede a la aplicación se ingresan los datos generales del deportista (Nombre, edad, documento de identificación, tipo de discapacidad, y clasificación funcional) y de la liga a la que pertenece. El número de identificación será el código con que el deportista queda registrado en la base de datos para realizar las consultas. Una vez registrados los datos generales del deportista, se ingresan los resultados obtenidos por el deportista en los campos de los fundamentos técnicos en todos los box y se guardan los datos. 


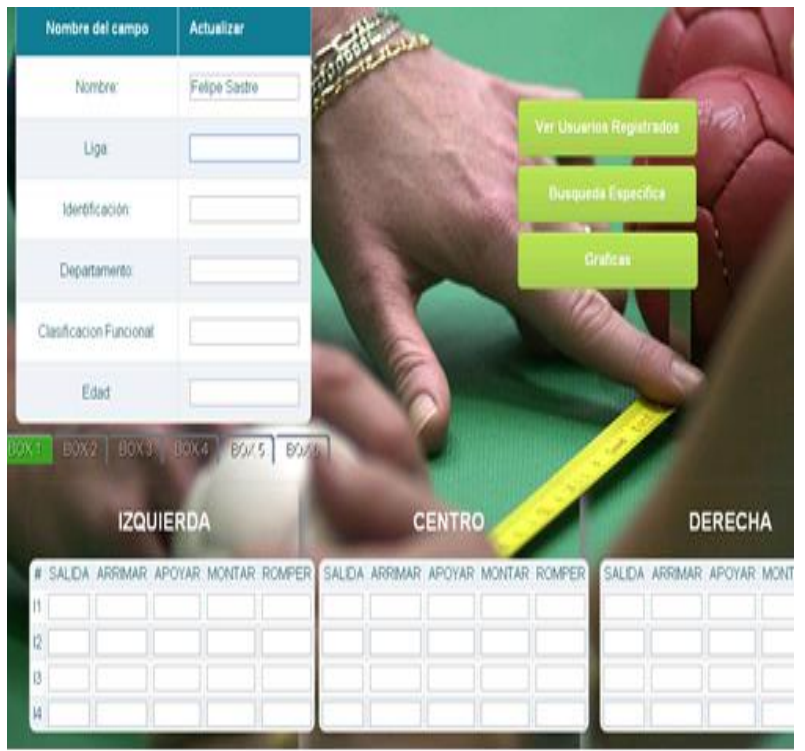

Figura 9. Ventana de registro datos generales y resultados del test.

A partir de la base de datos el programa puede generar un informe individual, en el campo de "Tabla de registro" se selecciona el box al cual se quiere acceder para observar los resultados obtenidos por el jugador, en cada uno de los fundamentos del lanzamiento, por zona y cuadrante de forma discriminada. En las figuras 10 y 11 se presenta un ejemplo
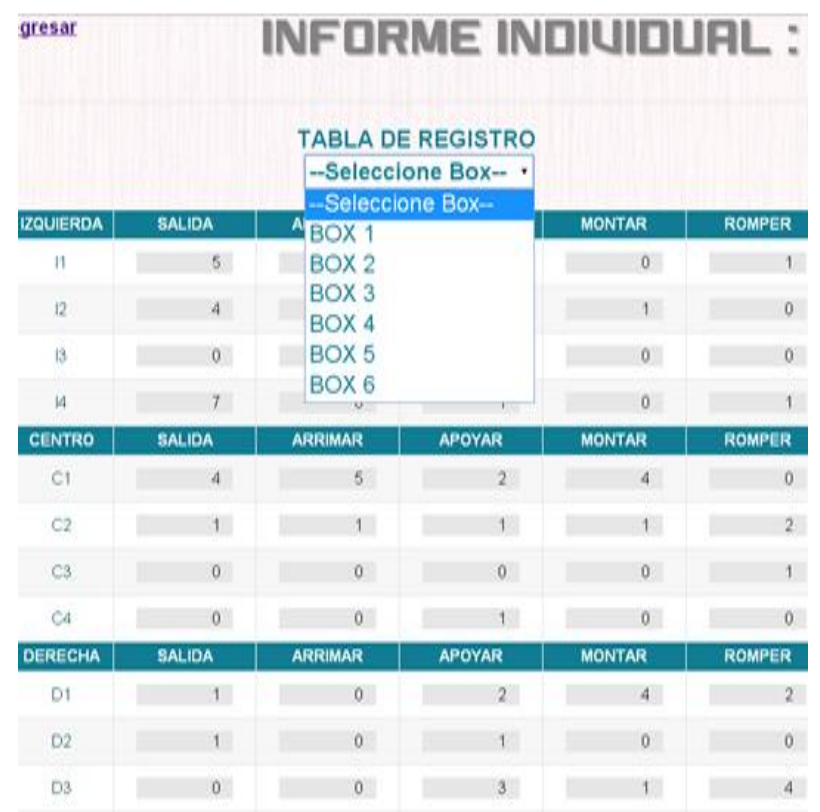

Figura 10. Reporte Individual
A continuación se muestra el registro de un deportista, en el costado derecho se observa en lo recuadros azules, el nivel en que se encuentra en cada fundamentos
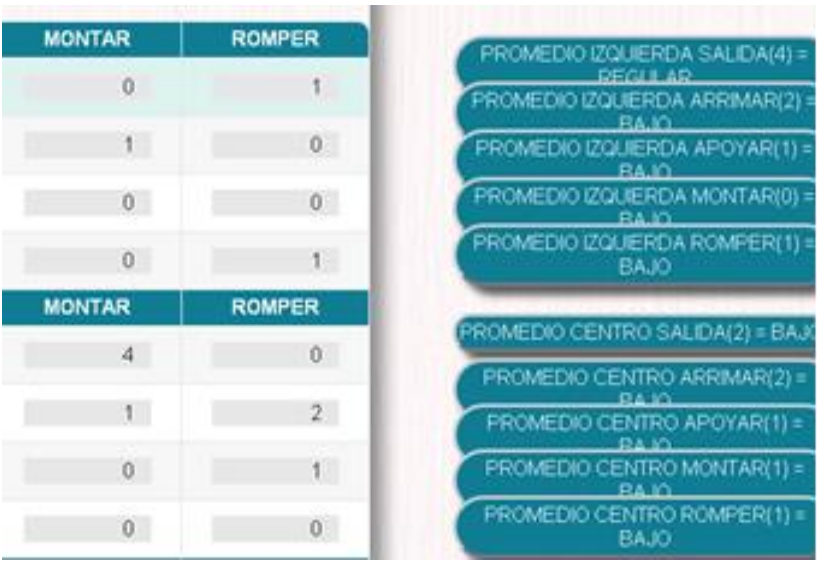

Figura 11. Ejemplo de reporte individual con calificación

La funcionalidad del software, permite analizar los resultados de cada jugador, sumando su desempeño en cada fundamento técnico, y también permite analizar el desempeño de todos los deportistas evaluados en cada uno de estos fundamentos, por lo que la información que aporta a los entrenadores es fundamental en los procesos de entrenamiento.

\section{DISCUSIÓN (O ANÁLISIS DE RESULTADOS)}

En la actualidad, es una necesidad evaluar el rendimiento deportivo de manera más objetiva, a partir de una sistematización eficiente de la información, de tal manera que facilite los procesos de feedback entre entrenadores $y$ deportistas, incidiendo de manera directa en mejores resultados en el entrenamiento y en las competencias, Bustamante y Burillo, [12]. Por esta razón el diseño del sistema de evaluación de la efectividad en el lanzamiento en Boccia se constituye en una herramienta fundamental para el desarrollo de un deporte que tiene una historia resiente en paralimpismo y que cuenta con un potencial de desarrollo importante a nivel nacional e internacional.

Los estudios relacionados con el análisis de juego en el deporte de Boccia enfocados a la evaluación y control de la técnica son escasos, el estudio de Molik, Zubala, Slyk, Bigas, Gryglewicz y 
Kucharczyk [17] analiza la motivación de los deportistas para la práctica de este deporte, en relación con su medio ambiente, teniendo en cuenta su edad, experiencia de formación e intensidad del entrenamiento, por su parte la investigación de Morriss y Wittmannová [18] evalúan los efectos de los horarios de entrenamiento de los deportistas de Boccia frente a su desempeño en actividades específicas de lanzamiento y de habilidades motrices.

Sirera, [19] analiza los segmentos corporales que intervienen en el lanzamiento y la fuerza, la velocidad del lanzamiento y la dirección, como elementos fundamentales en la adquisición de los aspectos técnicos tácticos en el desarrollo de la Boccia. Vega [20], se enfoca en el aumento de angulación de movilidad del brazo izquierdo de un deportista de Boccia con parálisis cerebral utilizando técnicas de electro estimulación y streching, dirigidas al mejoramiento de la técnica del lanzamiento. Calverol, [21] analiza la efectividad del lanzamiento, tomando como criterio únicamente la distancia entre la diana y la Boccia de color, evaluando la efectividad en la técnica según los ángulos del brazo y el balanceo que se realiza antes de soltar la bola. Esta metodología de medida empleada por Calverol, [21] se constituye en la base de trabajo para el presente estudio.

Por su parte Moreno, [22], se enfocó en el diseño y aplicación de un protocolo de evaluación de las habilidades técnicas utilizadas en el Boccia, en este test se consideró como criterios: la fuerza, la dirección y la precisión, en los 6 lanzamientos que cada participante realiza desde el box central. Aunque la investigadora no encontró resultados estadísticamente significativos, por usar una muestra reducida, si observo mejoras en el control de la dirección y en la reducción de la dispersión de las bolas.

En Colombia, solo se tiene evidencia de un test de precisión que evalúa todos los fundamentos técnicos, pero no se tiene registro de una investigación formal que utilice este test, (esta prueba, se está empleando actualmente a nivel nacional). En este test se divide el campo de juego en 7 zonas, y tiene como objetivo evaluar los fundamentos técnicos en las zonas establecidas. A partir de observaciones en su ejecución en competencias fundamentales, es posible considerar que este test puede generar una tendencia de juego, puesto que el deportista condiciona sus lanzamientos siempre a las mismas zonas.

El presente estudio, es pionero dentro del deporte del Boccia en diseñar un sistema integrado que incorpora la tecnología con el propósito de mejorar las condiciones de evaluación de la efectividad de los fundamentos del lanzamiento en Boccia. Entendiendo la efectividad, como el lanzamiento, que garantiza el logro de altos resultados deportivos, que son medibles, Morante, 2004, citado por García, [23], Este sistema le proporciona al entrenador herramientas para realizar un control objetivo del entrenamiento y la tendencia de juego de sus deportistas, al tiempo que le permite identificar sus debilidades y fortalezas, cuando juega de forma individual, en parejas, o en equipos.

Oña, Martínez, y Moreno, [24], consideran que el análisis de los parámetros que soportan el fundamento técnico de un deporte es una vía para mejorar el rendimiento, por lo tanto debe favorecerse a través del desarrollo de sistemas automáticos regulados por computador, favoreciendo como lo afirman, Carpentier y Geneviève, [25] una retroalimentación orientada a la promoción de la Información, los objetivos y funciones controlables de rendimiento. Estos procesos de retroalimentación posibilitan que el deportista, tenga expectativas altas pero realistas que servirán como parámetros a potencializar en el entrenamiento.

De igual manera el sistema de evaluación diseñado en el presente estudio logró determinar las zonas con más efectividad de juego de los deportistas y las zonas donde presenta mayor dificultad, permitiendo de esta manera la posibilidad a los entrenadores y a sus deportistas de crear esquemas de juego para las competiciones por parejas o por equipos.

Otro aporte fundamental e innovador del sistema de evaluación desarrollado, es que el deportista se obligado a jugar en las 12 zonas donde se lleva a cabo el juego, por lo que se soluciona el problema del condicionamiento de los lanzamientos, obligando al deportista a solucionar problemas y proponer jugadas en cualquier parte del terreno de juego, a diferencia de los test propuestos por Calverol [21], que puede generar una tendencia de juego, debido a que en su implementación puede condicionarse al deportista para que sus lanzamientos siempre vayan a la misma zona. 
En la última década se observa una incremento de profesionales de diversas áreas, interesados en el desarrollo de sofisticados dispositivos tecnológicos, que faciliten la práctica deportiva, esto se evidencia en la investigación de Morante y García [26] quienes desarrollan un software interactivo, diseñado como herramienta de ayuda para los entrenadores de Voleibol, igualmente ya se utilizan dispositivos para el análisis del rendimiento, como el diseñado por Alarcón, Urrutia y Callejeras [27] que permite capturar, controlar y monitorizar variables físicas relacionadas con la práctica del ciclismo, igualmente, estos avances se encuentran en el campo de la discapacidad donde a través del deportes como el Boccia se hace uso de la tecnología, como ejemplo de ello se puede referenciar el Boccia Game Simulator desarrollado por Machado, [28] el cual proporciona una experiencia virtual de juego, para novatos y deportistas. En este sentido, se ratifica la importancia de continuar con el desarrollo de sistemas de evaluación que incluyan dispositivos tecnológicos como Hardware y Software que contribuyan de manera eficiente en el trabajo de los entrenadores.

Igualmente la utilización de este tipo de sistema de evaluación y control de los lanzamientos del Boccia, permiten a los entrenadores y deportistas, adecuar sus esquemas de juego de acuerdo a tendencias tácticas empleadas a nivel internacional, y en la medida que se generalice su empleo en Colombia, se podrán tener estadísticas más precisas que muestren el nivel de desempeño de cada jugador o de cada equipo participante en Boccia.

\section{CONCLUSIONES}

El sistema integrado permite analizar la efectividad del lanzamiento de la Boccia, según la división de juego del deportista y su ubicación en cada box. Detectar las fortalezas y debilidades de juego del deportista y definir en cuál de los boxs el jugador demuestra un mejor desempeño, con el propósito de determinar su mejor ubicación en las competencias.

Esto le permitirá a los deportistas mejoras importantes en los aspectos técnicos del lanzamiento, al igual se proyecta que este sistema de evaluación sea empleado en todas las regiones del país brindándole a los entrenadores una herramienta de control, de igual manera se espera que la federación, pueda comenzar su implementación a nivel nacional, como una estrategia de mejora en las participaciones Internacionales de la Selección Colombia de Boccia.

\section{AGRADECIMIENTOS}

Agradecemos de manera especial, a los deportistas de Boccia de la selección de Cundinamarca, quienes hicieron posible el proceso de diseño y validación de este sistema de evaluación para la efectividad de los fundamentos técnicos del Boccia.

\section{REFERENCIAS BIBLIOGRÁFICAS}

[1] Ruiz, S. Deporte Paralímpico: Una mirada hacia el futuro. Futuro del paralimpismo. $\quad 15$, pp. 97104. 2012.

[2] Boccia International Federation. About Boccia. Recuperado de: http://www.bisfed.com/aboutboccia/. 2016.

[3] Rosell. C., Camats, E., Basil, C. Alumnado con discapacidad Motriz, Barcelona: Grao, 2010.

[4] Silva, C., Fonseca, D., Mateus, H., Contreras, N., y Restrepo, C. Distrofia muscular de Duchenne y Becker. Una visión molecular. Acta Médica Colombiana. 30 (3), pp. 112-116. 2005.

[5] Martin, D., Carl, K., y Lehnertz, k. Manuel de metodología del entrenamiento deportivo. Barcelona: Paidrotribo. 2001.

[6] Collazo, A. Metodología del Entrenamiento Deportivo. La Habana: Pueblo y Educación. 2007.

[7] Barrios, J., Ranzola, A. Manual para el deporte de iniciación y desarrollo. México: Ediciones deportivas Latinoamericanas. 1998.

[8] Harre, D, Teoría del entrenamiento deportivo. Buenos Aires: Editorial Stadium. 1983.

[9] Green, H. ¿Qué miden las pruebas? En. Mac Dougall, J., Wenger, H. y Green, H. Evaluación fisiológica del deportista. Barcelona: Paidrotribo, pp. 21-36. 1995.

[10] Avila, F., Moreno, F. La percepción y la actividad neuromuscular en personas con parálisis 
cerebral en el deporte de la Boccia. Una propuesta metodológica de valoración. Apunts educación física y deportes, (60), pp. 59 -62. 2000.

[11] Ramón, M., Ortega, C., Entrenamiento deportivo en relación con la función motora gruesa y la independencia funcional en niños y jóvenes con parálisis cerebral pertenecientes a la selección valle de Boccias. [Tesis de maestría]. Manizales, Universidad de Manizales, 2010.

[12] Bustamante, A., Burrillo, P. Gestión y evaluación del rendimiento en baloncesto: una revisión sistemática del software, Retos, 29, pp. 72 78. 2016.

[13] Manno, R., Fundamentos del entrenamiento deportivo. España: Paidotribo. 1994.

[14] Lozada, J., Casal, C., y Ardá, A. Como mejorar la efectividad en un jugador de tenis: modelos de regresión log-lineales, Cuadernos de psicología del deporte, 15, (1), pp. 63-70. 2015.

[15] Acero J. Conceptualización y Ámbito de la Biomecánica Instituto de Investigaciones y Soluciones Biomecánicas, Cali. Colombia. Recuperado de: http://g-se.com/es/org/ii-sb/blog.

[16] Acero, J. Aplicabilidad de la variabilidad en los análisis biomecánicos del gesto y el entrenamiento deportivo. En: Biomecánica deportiva y control del entrenamiento (Suarez, G.). Medellín: Funámbulos editores. pp. 45-84. 2009.

[17] Molik, B., Zubala, T., Słyk K,, Bigas, G., Gryglewicz, A., y Kucharczyk, B. Motivation of the disabled to participate in chosen Paralympics events (wheelchair basketball, wheelchair rugby, and Boccia). Physiotherapy, 18(1), pp. 42-51. 2010.

[18] Morriss, L.,Wittmannová, J. The effect of blocked versus random training schedules on Boccia skills performance in experienced athletes with cerebral palsy, European Journal of Adapted Physical Activity, 3(2), pp. 17- 28. 2010.

[19] Sirera, J. Aspectos técnicos tácticos en el desarrollo de la Boccia. Valencia. Disponible en: http://www.amicsdelaboccia.com/formacion/02_enc uentro_formativo/Aspectos_tecnicos_tacticos_en_b occia.pdf. 2011.
[20] Vega, A. Aumentar los grados de los ángulos de movilidad del brazo izquierdo en una persona con parálisis cerebral deportista de Boccia. [Tesis de pregrado]. Soacha, Universidad de Cundinamarca, 2013.

[21] Calverol, T. Estudio biomecánico de los lanzamientos de Boccia. [Tesis de Postgrado]. Catalunya, España, Universidad de Barcelona, 1999.

[22] Moreno, M. Boccia: Diseño y aplicación de un programa de evaluación y entrenamiento. [Trabajo de fin de grado en ciencias de la actividad física y del deporte. Facultad de Ciencias de la Actividad Física y el Deporte]. León, Universidad de León, 2015.

[23] García, O. Valoración de la eficacia del rendimiento deportivo en el lanzamiento del tejo, Revista Digital Educación Física y deportes, 16, (164), 2012.

[24] Oña, A. Martínez, M. y Moreno, F. Descripción de un sistema informatizado de procesamiento automático para la optimización del rendimiento deportivo basado en el control de la información, Motricidad European Journal of Human Movement, 1, pp. 45-56. 1994.

[25] Carpentier, J., y Geneviève, M. When changeoriented feedback enhances motivation, well-being and performance: A look at autonomy-supportive feedback in sport, Psychology of Sport and Exercise, 14, pp. 423-435. 2013.

[26] Morante, J., García, J. Entrenadores de voleibol, el software Voley Train. Kronos. Revista universitaria de la actividad física y el deporte, 2, pp. 20-24. 2003.

[27] Alarcón, A., Urrutia, J., Callejeras, M., Aplicación Móvil para la Administración de Variables Físicas en Ciclismo al Aire Libre. Inf. Tecnol, 27, 4, pp. 1-4. 2016.

[28] Machado, J. Boccia Game Simulator Applications for Training Cerebral Palsy Patients. [Tesis maestria, Mestrado Integrado em Engenharia Eletrotécnica e de Computadores]. Porto: Universidade do Porto, 2017. 